\title{
Publisher's Note: Dynamics and thermodynamics of systems with long-range dipole-type interactions [Phys. Rev. E 95, 022110 (2017)]
}

\author{
Boris Atenas and Sergio Curilef \\ (Received 30 March 2017; published 11 April 2017)
}

DOI: 10.1103/PhysRevE.95.049901

This paper was published online on 8 February 2017 with errors in Eqs. (4) and (5) and text on page 2. Equation (4) should read as

$$
\dot{p}_{i}=-\frac{\epsilon}{2}\left(2 M_{x} \sin \theta_{i}+M_{y} \cos \theta_{i}\right)
$$

Equation (5) should read as

$$
V=-N \frac{\epsilon}{2}\left(2 M_{x}^{2}-M_{y}^{2}+\Delta\right),
$$

The text following Eq. (5) on page 2 should read as "where $\Delta=\sum_{i, j} \Delta_{i, j}^{\prime}$ with $\Delta_{i, j}^{\prime}=\Delta_{i, j} / N^{2} \ldots$ " The paper has been corrected as of 29 March 2017. The equations and text are incorrect in the printed version of the journal. 